\title{
Compreensão da prescrição de medicamentos em uma unidade básica de saúde: autorrelato do usuário e aferição pelo pesquisador
}

\author{
Understanding of drug prescriptions in a basic health unit: patient self-report and \\ researcher assessment
}

\author{
Cristiane Olinda Coradi', Jussara dos Santos Cardoso ${ }^{2}$, Ronara Camila de Souza Groia ${ }^{3}$, Karina Cristina Lima Silva ${ }^{4}$, \\ Maria das Graças Braga Ceccato ${ }^{5}$, Marina Guimarães Lima ${ }^{5} \bowtie$ \\ 1 Programa de Pós-Graduação em Medicamentos e Assistência Farmacêutica da Universidade Federal de Minas Gerais (UFMG). Belo Horizonte, MG. \\ 2 Curso de Farmácia da UFMG. Belo Horizonte, MG. \\ ${ }^{3}$ Hospital das Clínicas da UFMG. Belo Horizonte, MG. \\ ${ }^{4}$ Hospital Risoleta Tolentino Neves. Belo Horizonte, MG \\ Departamento de Farmácia Social da Faculdade de Farmácia da UFMG. Belo Horizonte, MC.
}

\section{RESUMO}

Objetivos: Avaliar a compreensão das receitas de medicamentos por parte dos pacientes, comparando o autorrelato do usuário com a aferição do pesquisador.

Métodos: Um estudo transversal foi realizado no período de novembro de 2013 a fevereiro de 2014 em uma Unidade Básica de Saúde do município de Belo Horizonte, Minas Gerais. Foram entrevistados os usuários com 18 anos de idade ou mais que buscaram medicamentos na farmácia da unidade, para si ou para menores sob sua responsabilidade. A seleção da amostra foi por conveniência, não probabilística. Durante a entrevista, os usuários declaravam se haviam entendido os seguintes dados das prescrições: nome do medicamento, dose e frequência. Posteriormente, os participantes repetiam para os pesquisadores os dados das receitas, podendo consultá-las no mesmo momento. Após as entrevistas, as respostas às perguntas foram interpretadas pelos pesquisadores, que avaliaram a concordância entre as informações fornecidas pelos usuários e as reais instruções das receitas, utilizando o teste de Kappa.

Resultados: Foram coletados dados de 69 usuários da farmácia da unidade (correspondentes a 69 receitas dispensadas). Cinquenta e nove $(85,5 \%)$ usuários relataram ter compreendido todos os dados das prescrições (autorrelato), enquanto segundo a aferição dos pesquisadores, foram $23(33,3 \%)$ entrevistados que compreenderam simultaneamente todos os dados, referentes ao nome do medicamento, número de doses diárias e horário de administração. O teste de Kappa indicou um grau de concordância entre o autorrelato e a aferição dos pesquisadores de 0,138 (ligeira) considerando todos os itens da receita, sendo que especificamente sobre o número de doses a concordância foi de - 0,055 (fraca). Conclusões: A taxa de compreensão das prescrições de medicamentos autorrelatada pelos pacientes não concordou com a avaliação dos pesquisadores, a qual indicou uma taxa de compreensão menor do que a autorrelatada. Este resultado sugere que o autorrelato dos pacientes com relação à compreensão da prescrição apresenta limitações e deve ser interpretado com cautela na pesquisa e na prática clínica. Recomenda-se que sejam aprimorados os meios de fornecer a informação ao paciente a fim de contribuir para a compreensão adequada da prescrição de medicamentos. DESCRITORES: assistência farmacêutica; atenção primária à saúde; avaliação em saúde; farmacoterapia.

\section{ABSTRACT}

Aims: To evaluate the understanding of drug prescriptions by patients, comparing the user self-report with the researcher assessment.

Methods: A cross-sectional study was conducted from November 2013 to February 2014 at a Basic Health Unit in the city of Belo Horizonte, Minas Gerais, Brazil. Users 18 years of age or older who sought medicines in the unit's pharmacy, for themselves or for minors under their care, were interviewed. The selection of the sample was non-probabilistic, for convenience. During the interview, the users informed if they had understood the following data of the prescriptions: drug name, dose and frequency of administration. Participants then repeated the data for researchers, being allowed to check the prescriptions at the same time. After the interviews, the answers to the questions were interpreted by the researchers, who evaluated the agreement between the information provided by the users and the actual instructions of the recipes, using the Kappa test.

Results: Data were collected from 69 users of the unit's pharmacy (corresponding to 69 delivered prescriptions). Fifty-nine (85.5\%) users reported having understood all the prescriptions data (self-report), while according to the measurement of the researchers, 23 (33.3\%) were the respondents who simultaneously understood all data, relating to the name of the drug, number of daily doses and schedule. Kappa test indicated a degree of agreement between self-report and measurement of researchers of 0.138 (slight) considering all items of the prescription, and specifically on the number of doses the agreement was -0.055 (poor).

Conclusions: Self-reported rate of patient's understanding of drug prescriptions was higher than the assessed by the researchers. This result suggests that self-report of patients with respect to the understanding of prescriptions has limitations and should be interpreted with caution both in research and in clinical practice. It is recommended to enhance the means of providing information to patients, in order to contribute to the proper understanding of drug prescriptions.

KEY WORDS: pharmaceutical services; primary health care; health evaluation; drug therapy. 
Abreviaturas: UBS, unidade básica de saúde.

\section{INTRODUÇÃO}

O medicamento apresenta função relevante na proteção e recuperação da saúde. Para tanto, é necessário o seu uso racional, caso contrário poderão ocorrer sérios danos à saúde, além de possibilitar o mascaramento e agravamento de doenças [1,2]. O uso racional de medicamentos, segundo a Política Nacional de Medicamentos, compreende "a prescrição apropriada, a disponibilidade oportuna a preços acessíveis, a dispensação em condições adequadas, o consumo nas doses indicadas, nos intervalos definidos e no período de tempo indicado de medicamentos eficazes, seguros e de qualidade" [3]. Neste contexto, a compreensão da prescrição é uma condição fundamental para o uso racional dos medicamentos. A não compreensão da terapia medicamentosa pelos pacientes e das informações a eles prestadas também contribui para a não adesão e pode trazer insucesso terapêutico, aumento de eventos adversos, automedicação e agravamento do estado clínico, com consequente piora da qualidade de vida [4,5].

Na promoção do uso racional de medicamentos, um dos pontos importantes é a avaliação da compreensão do usuário a respeito da farmacoterapia prescrita. Estudos com este mesmo foco já mostraram um conhecimento insuficiente dos pacientes em relação aos medicamentos prescritos [6-9]. Entretanto, a abordagem sobre a compreensão da prescrição pelos usuários de medicamentos na Atenção Primária à Saúde ainda é escassa na literatura. Além disso, os estudos avaliam geralmente a compreensão relatada pelo usuário, e não a aferida pelo pesquisador. Esse tipo de levantamento é importante para redirecionar ações de Assistência Farmacêutica de maneira a que contribuam mais efetivamente com a promoção do uso racional de medicamentos.

Dessa forma, foi delineado este estudo, que avaliou a compreensão dos usuários da farmácia de uma Unidade Básica de Saúde (UBS) do município de Belo Horizonte, Minas Gerais, sobre a prescrição de medicamentos, comparando o ponto de vista do próprio usuário com a aferição do pesquisador.

\section{MÉTODOS}

Foi realizado um estudo transversal em uma UBS do município de Belo Horizonte, Minas Gerais, que atende a uma área de aproximadamente 25.000 habitantes. O projeto foi aprovado pelo Comitê de Ética em Pesquisa da Universidade Federal de Minas Gerais sob o parecer de número 307.396 de 13/06/2013 e pelo Comitê de Ética em Pesquisa da Secretaria Municipal de Saúde de Belo Horizonte sob o parecer de número 601.089-0 de 13/08/2013. Com a finalidade de preservar a confidencialidade dos dados, não consta no artigo o nome da unidade de saúde e os funcionários não foram identificados nas entrevistas.

Foram incluídos na entrevista usuários com 18 anos de idade ou mais, que buscavam por medicamentos na farmácia da UBS para eles próprios ou para menores sob sua guarda. As entrevistas foram realizadas com os usuários que concordassem em participar da pesquisa e assinassem o Termo de Consentimento Livre e Esclarecido. A seleção da amostra foi por conveniência, não probabilística. Definiu-se previamente que as entrevistas com os usuários seriam realizadas no período de novembro de 2013 a fevereiro de 2014, três vezes por semana. Buscou-se entrevistar todos os pacientes que buscavam por medicamentos na farmácia na UBS nesse período e dias determinados. A coleta de dados foi realizada por uma equipe composta pela pesquisadora responsável pelo estudo e por três estudantes envolvidos no Programa de Educação pelo Trabalho para a Saúde-PET-Saúde III. Foram realizadas entrevistas com os participantes e coletados dados referentes às informações observadas pelos pesquisadores nas prescrições. O formulário utilizado foi baseado em indicadores propostos por uma publicação da Organização Mundial de Saúde [10] e adaptados para o contexto específico de uma UBS. Foi composto por itens referentes às características sociodemográficas dos usuários e perguntas sobre as prescrições e os medicamentos prescritos.

Um manual sobre coleta de dados foi elaborado com o objetivo de uniformizar os procedimentos adotados pelos pesquisadores. Previamente à coleta de dados foi realizado um teste piloto por um mês para que fossem realizadas adaptações nos instrumentos. Nessa etapa, a concordância entre os pesquisadores acerca dos dados dos formulários preenchidos foi avaliada pelo teste de Kappa e todos os valores encontrados foram superiores a 0,90 indicando excelente concordância entre os pesquisadores.

Para a descrição da legibilidade das prescrições, consideraram-se prescrições legíveis aquelas que não implicaram em dificuldades na leitura de todos os seus dados, de forma a não gerar dúvidas para o entendimento dos pesquisadores e dos entrevistados. Adicionalmente foi analisada a frequência de 
prescrições com dados completos (legibilidade, posologia, horário de administração).

O nível de compreensão dos participantes sobre os medicamentos prescritos foi avaliado com base nas propostas da Organização Mundial de Saúde [10] por meio de perguntas relacionadas aos itens nome, dose e frequência de administração do medicamento. Foi analisada a receita que o participante apresentava em mãos no momento da entrevista, não sendo avaliados medicamentos que estivessem sendo utilizados por automedicação. No momento da entrevista, foi permitido ao indivíduo consultar a receita. Considerou-se, para a análise da compreensão, todos os medicamentos prescritos.

Inicialmente, a compreensão da prescrição foi avaliada segundo o autorrelato do participante para cada item, por meio de respostas dicotômicas ( $\operatorname{sim} /$ não). Os usuários relatavam se haviam entendido os seguintes dados das prescrições: nome do medicamento, dose e frequência. Posteriormente, consultando a receita, os participantes informavam aos pesquisadores qual era o nome do medicamento, dose e frequência de administração. Os pesquisadores transcreviam as respostas fornecidas pelos pacientes e os dados das prescrições. Após as entrevistas, as respostas às perguntas eram interpretadas pelos pesquisadores, que avaliavam se as informações fornecidas pelo paciente concordavam com as que estavam nas instruções das receitas.

$\mathrm{Na}$ avaliação da concordância entre os dados relatados pelos usuários e os dados das prescrições, os pesquisadores realizaram considerações para situações especiais. Por exemplo, se a prescrição continha a posologia de administração às 10 horas e o paciente relatou que o medicamento devia ser usado de manhã, considerou-se que ele compreendeu a posologia e sua resposta estava certa. Erros de pronúncia do nome do medicamento não foram considerados errados. Quando o paciente relatava a cor do produto farmacêutico, mas não sabia relatar o nome, considerou-se que ele não havia compreendido o nome do medicamento.

$\mathrm{Na}$ análise de dados, considerou-se que o usuário compreendeu integralmente a prescrição se os dados sobre nome, dose e frequência estivessem corretos simultaneamente. A unidade de análise foi o participante, para a análise da compreensão. A análise de dados incluiu inicialmente a estatística descritiva, com medidas de tendência central (média e mediana) e medidas de variação (desvio padrão) para as variáveis quantitativas. O nível de concordância entre os dados relatados pelos usuários e os aferidos pelos pesquisadores foi calculado pelo Índice Kappa, utilizando-se a escala de Landis e Koch, que considera como graus de concordância $<0,0$ (fraca), 0 a 0,20 (ligeira), 0,21 a 0,40 (razoável), 0,41 a 0,60 (moderada), 0,61 a 0,8 (substancial) e 0,81 a 1 (excelente). Os dados foram analisados no software GNU PSPP versão 0.8.2-gc473f9.

\section{RESULTADOS}

Foram incluídos 69 usuários que atenderam aos critérios de inclusão, totalizando 69 prescrições e 215 medicamentos. Quinze (21,7\%) usuários tinham 60 anos ou mais. Quanto às características das prescrições, o número de medicamentos prescritos variou de um a nove, com média de $3,1 \pm 1,9$ e mediana de 3. Dentre as 69 prescrições, em 67 (97\%) estava descrita a posologia. Sessenta e cinco receitas $(94,2 \%)$ foram consideradas legíveis. Em 11 (16\%) prescrições não havia o horário de administração (Tabela 1).

Tabela 1. Avaliação das prescrições de medicamentos para usuários atendidos em uma Unidade Básica de Saúde de Belo Horizonte segundo critérios selecionados, Minas Gerais, $2013(n=69)$

\begin{tabular}{lc}
\hline \multicolumn{1}{c}{ Critérios } & $\begin{array}{c}\text { Presença na prescrição } \\
\text { n (\%) }\end{array}$ \\
\hline Legibilidade & $65(94,2)$ \\
Posologia & $67(97,1)$ \\
Horário de administração & $58(84,1)$ \\
\hline
\end{tabular}

Quanto à compreensão das prescrições segundo o autorrelato dos participantes, observou-se que 67 $(97,1 \%)$ relataram entender o número das doses diárias prescritas e $65(94,2 \%)$ os horários de administração dos medicamentos. Sete participantes $(10,1 \%)$ relataram que não conheciam ou não sabiam ler o nome dos medicamentos prescritos (Tabela 2).

Em relação à compreensão das prescrições segundo avaliação pelo pesquisador, observaram-se diferenças na comparação entre a proporção desta e a proporção da autorrelatada pelo usuário. A compreensão simultânea dos três critérios (nomes dos medicamentos, número de doses diárias e horário de administração) foi autorrelatada por 59 (85,5\%) usuários, enquanto a avaliação dos pesquisadores observou que $23(33,3 \%)$ deles compreenderam simultaneamente os três critérios. Logo, o autorrelato apresentou proporção quase três vezes maior que a aferida pelos pesquisadores. Foi possível observar que a autopercepção do conhecimento foi maior para todos os itens analisados, como nome do medicamento, número de doses diárias e horário de administração. 
Tabela 2. Concordância entre a compreensão das prescrições segundo autorrelato dos usuários de uma unidade básica de saúde e a análise do pesquisador. Belo Horizonte, Minas Gerais, 2013 (n=69).

\begin{tabular}{|c|c|c|c|c|c|}
\hline \multirow{2}{*}{ Itens de compreensão da prescrição } & \multicolumn{2}{|c|}{ Autorrelato do usuário } & \multicolumn{2}{|c|}{ Análise do pesquisador } & \multirow{2}{*}{ Índice Kappa* } \\
\hline & $\mathbf{n}$ & $(\%)$ & n & $(\%)$ & \\
\hline \multicolumn{6}{|l|}{ Conhece ou sabe ler o nome dos medicamentos } \\
\hline Sim & 59 & $(85,5)$ & 37 & $(53,6)$ & \multirow{2}{*}{0,173} \\
\hline Não & 7 & $(10,1)$ & 32 & $(46,4)$ & \\
\hline \multicolumn{6}{|l|}{ Conhece o número de doses diárias prescritas } \\
\hline $\operatorname{Sim}$ & 67 & $(97,1)$ & 50 & $(72,5)$ & \multirow{2}{*}{$-0,055$} \\
\hline Não & 2 & $(2,9)$ & 19 & $(27,5)$ & \\
\hline \multicolumn{6}{|l|}{ Conhece os horários de administração } \\
\hline Sim & 65 & $(94,2)$ & 54 & $(78,3)$ & \multirow{2}{*}{0,015} \\
\hline Não & 1 & $(1,5)$ & 15 & $(21,7)$ & \\
\hline \multicolumn{6}{|l|}{$\begin{array}{l}\text { Compreende completamente a prescrição } \\
\text { (atende aos três critérios acima simultaneamente) }\end{array}$} \\
\hline Sim & 59 & $(85,5)$ & 23 & $(33,3)$ & \multirow{2}{*}{0,138} \\
\hline Não & 10 & $(14,5)$ & 46 & $(66,7)$ & \\
\hline
\end{tabular}

* Graus de concordância pela escala de Landis e Koch: $<0,0=$ fraca; 0-0,20=ligeira; 0,21-0,40=razoável;

$0,41-0,60=$ moderada, $0,61-0,8=$ substancial, $0,81-1=$ excelente.

O teste de Kappa indicou um grau de concordância entre o autorrelato e a aferição dos pesquisadores de 0,138 (ligeira) considerando todos os itens da receita, sendo que especificamente sobre o número de doses a concordância foi de $-0,055$ (fraca) (Tabela 2). Observou-se ainda que 15 entrevistados $(21,7 \%)$ não compreenderam nenhum dos critérios avaliados.

\section{DISCUSSÃO}

Esta pesquisa apresenta como potencialidade a divulgação da compreensão dos usuários da Atenção Primária à Saúde sobre a prescrição de medicamentos, conforme aferida pelos pesquisadores, e não apenas como autorrelato. A aferição do pesquisador busca identificar se a compreensão autorrelatada condiz com a realidade, observando a questão da segurança do paciente ao realizar a farmacoterapia no domicílio. Este ponto não tem sido suficientemente abordado nos estudos realizados sobre o assunto $[6,9,11,12]$.

Quanto ao número de medicamentos prescritos, o valor médio encontrado foi superior ao encontrado por estudos anteriores [4,5]. Este resultado pode sugerir polifarmacoterapia nesta amostra, podendo ser justificada pela frequência de idosos (em torno de um quinto) na amostra em estudo, uma vez que o uso de vários medicamentos é uma característica dessa faixa etária [13]. Muitos medicamentos prescritos ao mesmo tempo podem interferir com o entendimento do paciente, pois estudos publicados sugerem que quando há menos medicamentos a serem administrados, também há menos informações a serem assimiladas, facilitando a compreensão dos usuários $[4,8,14]$.

Observou-se que a proporção de prescrições legíveis e com presença de posologia foi superior à relatada por outros estudos [15-19], sugerindo maior adesão dos prescritores identificados no presente estudo às normas legais para a prescrição [20]. A completude e legibilidade da prescrição que propõe o esquema farmacoterapêutico são requisitos básicos para a compreensão do usuário acerca da prescrição a ele expedida. Essa compreensão é importante para o cumprimento adequado do tratamento farmacológico proposto e o uso racional do medicamento [14,21].

Os entrevistados conheciam mais as doses e os horários de administração do que o nome do medicamento a ser administrado. Esses achados podem indicar um risco na rotina dos usuários dos medicamentos. Pode acontecer uma troca quando se faz uso de mais de um medicamento [22], pois o usuário sabe qual quantidade e frequência de uso, mas não conhece o nome do medicamento a ser usado.

A proporção de entrevistados que demonstraram, após análise do pesquisador, não compreender completamente as suas prescrições foi de $66,7 \%$. Em um estudo realizado por Silva et al. [6], 31\% dos entrevistados não acertaram o nome do medicamento prescrito; $19 \%$ erraram a dose; e $31 \%$, a frequência da administração prescrita. Em estudo semelhante, realizado no Distrito Federal em 2001, 80\% dos pacientes não compreendiam qual medicamento havia sido prescrito ou a forma correta de administrá-lo [11]. 
Dos Santos e Nitrini [12] obtiveram que o indicador de percentagem de pacientes que não conheciam a dose correta foi de $30 \%$. Uma pesquisa realizada em um hospital universitário no Paquistão apontou que, dentre os pacientes sem escolaridade formal, $50 \%$ não compreendiam a dose do medicamento e $56 \%$ não entenderam qual era o horário de administração e a duração do tratamento [9].

Os resultados apontam para o preocupante fato de que os usuários entrevistados podiam não ter condições de realizar a farmacoterapia prescrita com segurança. Se considerarmos o fato de que muitos desses medicamentos foram destinados ao tratamento de doenças crônicas, era de se esperar um maior entendimento das prescrições. Também se deve notar que a todos os entrevistados foi permitido consultar a prescrição para responder aos questionamentos e que estas se mostraram satisfatoriamente legíveis. Portanto, o percentual de pacientes que não compreenderam a prescrição é considerável. Falhas na compreensão podem acarretar falhas na terapia e podem ser evitadas. Em função disso, é importante a intervenção ativa dos profissionais de saúde para garantir a compreensão dos pacientes acerca da farmacoterapia prescrita no momento da consulta e por ocasião da dispensação dos medicamentos.

Alguns métodos são citados em bibliografia recente com o intuito de capacitar profissionais dispensadores. Por exemplo, para auxiliar os pacientes no uso correto dos medicamentos prescritos, os profissionais de saúde, em especial o farmacêutico dispensador, pode elaborar uma tabela de aprazamento da medicação de forma simples e até pictografada. Esse método pode contribuir para o entendimento da prescrição, aumentando a segurança do indivíduo no processo farmacoterapêutico, e deve fazer parte da rotina farmacêutica. Além disso, faz-se necessária a capacitação de profissionais tanto prescritores quanto dispensadores, assim como disponibilidade de tempo suficiente para a orientação conforme a necessidade de cada paciente, além da conscientização sobre a importância da informação no tratamento farmacoterapêutico $[23,24]$.

Este estudo apresenta limitações, como seleção da amostra por conveniência. Além disso, poderiam ter sido usados outros critérios de avaliação da compreensão dos usuários sobre a prescrição, como tempo de tratamento, indicação, reações adversas e interações medicamentosas.

Como ponto positivo, esta pesquisa apresenta a divulgação de informações sobre compreensão do tratamento por usuários de uma UBS da Atenção Primária à Saúde no Sistema Único de Saúde, avaliada por comparação com os dados presentes nas receitas. Este tema é abordado com insuficiência na literatura, que geralmente não apresenta a análise do pesquisador sobre os dados relatados, o que foi feito no presente estudo.

Diante dos resultados obtidos, conclui-se que a taxa de compreensão das prescrições de medicamentos autorrelatada pelos pacientes não concordou com a avaliação dos pesquisadores. A grande maioria dos entrevistados não possuía bom nível de compreensão sobre os medicamentos prescritos, entretanto em geral não tinham consciência dessa limitação, já que a taxa de compreensão autorrelatada foi superior à avaliada pelos pesquisadores. Este resultado sugere que o autorrelato dos pacientes com relação à compreensão da prescrição apresenta limitações e deve ser interpretado com cautela na pesquisa e na prática clínica. Recomenda-se que sejam aprimorados os meios de fornecer a informação ao paciente a fim de contribuir para a compreensão adequada da prescrição de medicamentos.

\section{NOTAS}

Apoio financeiro

Este estudo obteve apoio do Ministério da Saúde por meio de bolsas concedidas a estudantes de graduação e docentes no contexto do Programa de Educação pelo Trabalho para a Saúde - PET-Saúde III.

Declaração de conflitos de interesse

Os autores declaram não haver conflitos de interesse relevantes ao conteúdo deste estudo.

\section{REFERÊNCIAS}

1. Aquino DS. Por que o uso racional de medicamentos deve ser uma prioridade? Cien Saude Colet. 2008;13(Supl):733-36. http://dx.doi, org/10.1590/S1413-8123200800070002

2. Portela AS. Prescrição médica: orientações adequadas para o uso de medicamentos? Cien Saude Colet. 2010;5(Supl 3):3523-28. http:// dx.doi.org/10.1590/S1413-81232010000900027 
3. Brasil. Ministério da Saúde. Política Nacional de Medicamentos. Brasília: Ministério da Saúde; 2001.

4. Cruzeta APS, Dourado ACL, Monteiro MTM, Martins RO, Calegario TA, Galato D. Fatores associados à compreensão da prescrição médica no Sistema Único de Saúde de um município do Sul do Brasil. Cien Saude Colet. 2013;18(12):3731-37. http://dx.doi.org/10.1590/ S1413-81232013001200029

5. Oenning D, Volpato de Oliveira B, Blatt CR. Conhecimento dos pacientes sobre os medicamentos prescritos após consulta médica e dispensação. Cien Saude Colet. 2011;16(7):3277-83. http://dx.doi.org/10.1590/S1413-81232011000800027

6. Sano PY, Masotti RR, Santos AAC, Cordeiro JA. Avaliação do nível de compreensão da prescrição pediátrica. J Pediatr (Rio J). 2002;78(2):140-45. http://dx.doi.org/10.1590/s0021-75572002000200013

7. Gimenes HT, Zanetti ML, Otero LM, Teixeira CRS. O conhecimento do paciente diabético tipo 2 acerca dos antidiabéticos orais. Cien Cuid Saude. 2006;5(3):317-25.

8. Fröhlich SE, Dal Pizzol TS, Mengue SS. Instrumento para avaliação do nível de conhecimento da prescrição na atenção primária. Rev Saude Pub. 2010;44(6):1046-54. http://dx.doi.org/10.1590/S0034-89102010000600009

9. Patel MJ, Khan MS, Ali F, Kazmi Z, Riaz T, Awan S, Sorathia AL. Patients' Insight of Interpreting Prescriptions and Drug Labels-A Cross Sectional Study. PLoS One. 2013;8(6):1-6. http://dx.doi.org/10.1371/journal.pone.0065019

10. World Health Organization. Operational package for assessing, monitoring and evaluating country pharmaceutical situations. Guide for coordinators and data collectors. Geneva: WHO; 2007.

11. Naves JOS, Silver LD. Avaliação da assistência farmacêutica na atenção primária no Distrito Federal. Rev Saude Publica. 2006;39(2): 223-30. http://dx.doi.org/10.1590/S0034-89102005000200013

12. Dos Santos V, Nitrini SMO. Indicadores do uso de medicamentos prescritos e de assistência ao paciente de serviços de saúde. Rev Saude Publica. 2004;38(6):819-26. http://dx.doi.org/10.1590/S0034-89102004000600010

13. Rosa GR, Camargo EAF. Polimedicação em idosos. Interciência \& Sociedade. 2014;3(2):72-8.

14. Acúrcio FA, Silva AL, Ribeiro AQ, Rocha NP, Silveira MR, Klein CH, Rozenfeld S. Complexidade do regime terapêutico prescrito para idosos, Belo Horizonte, MG. Rev Assoc Med Bras. 2009;55(4):468-74. http://dx.doi.org/10.1590/S0104-42302009000400025

15. Silvério MS, Leite ICG. Qualidade das prescrições em município de Minas Gerais: uma abordagem farmacoepidemiológica. Rev Assoc Med Bras. 2010;56(6):675-80. http://dx.doi.org/10.1590/S0104-42302010000600016

16. Ferreira VOG, Melnikov P, Toffoli-Kadri MC. Nível de entendimento de prescrições medicamentosas pediátricas em Unidades Básicas de Saúde. Rev Bras Saúde Matern Infant. 2011;11(3):249-56.

17. Oliveira LCF, Assis MMA, Barboni AR. Assistência Farmacêutica no Sistema Único de Saúde: da Política Nacional de Medicamentos à Atenção Básica à Saúde. Cien Saude Colet. 2010;15(Supl. 3):3561-67. http://dx.doi.org/10.1590/S1413-81232010000900031

18. Silva ERB, Bandeira VAC, Oliveira KR. Avaliação das prescrições dispensadas em uma farmácia comunitária no município de São Luiz Gonzaga - RS. Rev Ciênc Farm Básica Apl. 2012;33(2):275-81.

19. Ev LS, Guimarães AG, Castro VS. Avaliação das prescrições dispensadas em uma Unidade Básica de Saúde do Município de Ouro Preto, Minas Gerais, Brasil. Lat Am J Pharm. 2008;27(4):543-7.

20. Brasil. Lei $\mathrm{n}^{\circ}$ 5.991, de 17 de dezembro de 1973. Dispõe sobre o controle sanitário do comércio de drogas, medicamentos, insumos farmacêuticos e correlatos, e dá outras providências. Brasília, DF, 1973.

21. Lopes LN, Garcia KP, Dias LG, Soares LR, Leite AM, Silva JAC. Qualidade das prescrições médicas em um Centro de Saúde Escola da Amazônia Brasileira. Rev Soc Bras Clin Med. 2014;12(2):1-5.

22. Silva AEB, Reis AMM, Miasso AI, Santos JO, Cassiane SHB. Eventos adversos a medicamentos em um hospital sentinela do Estado de Goiás, Brasil. Rev Latino-Am Enf. 2011;19(2):1-9.

23. Brasil. Ministério da Saúde. Secretaria de Ciência, Tecnologia e Insumos Estratégicos. Departamento de Assistência Farmacêutica e Insumos Estratégicos. Cuidado Farmacêutico na Atenção Básica. 1ํa ed. rev. Brasília: Ministério da Saúde, 2015.

24. Menezes APS, Domingues MR, Baisch ALM. Compreensão das prescrições pediátricas de antimicrobianos em Unidades de Saúde em um município do sul do Brasil. Rev Bras Epidemiol. 2009;12(3):478-89. http://dx.doi.org/10.1590/S1415-790X2009000300016 